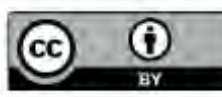

\title{
Caracterización de la tendencia del COVID-19 en Colombia con regresiones polinomiales*
}

\section{Characterization of the COVID-19 trend in Colombia with polynomial regressions}

\section{Caracterização da tendência de COVID-19 na Colômbia com regressões polinomiais}

Recibido: 30 de Noviembre de 2020. Aceptado: 16 de Marzo de 2021.

Publicado: 30 de diciembre de 2021.

DOI: https://doi.org/10.11144/Javeriana.rgps20-41.ctcc

\author{
Ariel Emilio Cortés Martínez \\ Pontificia Universidad Javeriana, Colombia \\ ORCID: https://orcid.org/0000-0002-1169-9086 \\ Carmen Elisa Becerra Huertas \\ Universidad Colegio Mayor de Cundinamarca, Colombia \\ ORCID: https://orcid.org/0000-0002-4325-652X
}

\begin{abstract}
Para citar este artículo: Cortés AE, Becerra CE. Caracterización de la tendencia del COVID-19 en Colombia con regresiones polinomiales. Rev Gerenc Polit Salud. 2021;20(41). https://doi.org/10.11144/ Javeriana.rgps20-41.ctcc
\end{abstract}

* Artículo de investigación 


\section{Resumen}

Objetivo. Desarrollar una serie de modelos polinomiales para seguir el comportamiento del crecimiento y tendencia de la curva de contagios y muertes por COVID-19 en Colombia. Métodos. Se utilizó la recolección diaria de los datos informados de contagiados confirmados y fallecidos diarios por COVID-19 entre el 06 de marzo y el 10 de abril de 2021. Para el análisis de predicción se utilizaron funciones polinomiales desarrolladas en Excel. Resultados. De las seis funciones polinomiales evaluadas el polinomio con mayor nivel de determinación es el de grado 6 según el R. ajustado. Se realizaron predicciones teniendo en cuenta las funciones polinomiales acumuladas de contagiados confirmados y fallecidos. Conclusión. Los modelos de fácil construcción en Excel como las funciones polinomiales, son útiles para la vigilancia de eventos de salud pública facilitando la toma de decisiones oportunas.

Palabras clave: Infecciones por coronavirus, epidemiologia, pandemias, transmisión, síndrome respiratorio agudo grave.

\section{Abstract}

Objective. To develop a series of polynomial models to track the growth and trend of infection and death curve for COVID-19 in Colombia. Methods. The infected and daily deaths from COVID-19 between March 6 to April 10, 2021 , were used. For its prediction analysis, we use polynomial functions in Excel. Results. Of the six polynomial functions evaluated, the polynomial with the highest level of determination is that of degree 6 according to the adjusted R.. Predictions were made taking into account the accumulated polynomial functions of confirmed infected and deceased. Conclusions. Easy-to-build Excel models such as polynomial functions are effective for monitoring public health events, facilitating timely decision-making.

Keywords: Coronavirus infections, epidemiology, pandemics, transmission, severe acute respiratory syndrome.

\section{Resumo}

Objetivo. Desenvolver uma série de modelos polinomiais para o comportamento de crescimento e tendência da curva de infecção e morte por COVID-19 na Colômbia. Métodos. Foi utilizada a coleta diária dos dados relatados de infecção confirmada e óbitos diários da COVID-19 entre 6 de março e 10 de abril de 2021. Para sua análise de predição, foram utilizadas funções polinomiais desenvolvidas em Excel. Resultados. Das 6 funções polinomiais avaliadas, o polinômio com maior nível de determinação é o de grau 6 de acordo com o R. ajustado. As previsões foram feitas levando em consideração as funções polinomiais acumuladas de infectados confirmados e falecidos. Conclusão. Modelos Excel fáceis de construir, como funções polinomiais, são úteis para monitorar eventos de saúde pública, facilitando a tomada de decisão oportuna.

Palavras-chave: Infecções por coronavírus, epidemiologia, pandemias, transmissão, síndrome respiratória aguda grave. 
En diciembre de 2019 se presentaron los primeros casos de neumonía atípica de origen desconocido en Wuhan, provincia de Hubei en China $(1,2)$. El patógeno fue un coronarovirus relacionado con el síndrome respiratorio de Oriente Medio (MERS-CoV) y el Síndrome respiratorio agudo severo (SARS-CoV). Este nuevo coronarovirus, denominado inicialmente SARS-CoV2 y más conocido en la enfermedad como COVID-19, se extendió por todos los continentes y el 11 de marzo de 2020 fue declarado pandemia por parte de la Organización Mundial de la Salud (OMS).

En Colombia, el primer caso autóctono se registró el 09 de marzo y el primer fallecido el 16 de marzo de 2020. A partir del 25 de marzo el Gobierno Nacional decretó una cuarentena obligatoria en todo el territorio hasta el 31 de agosto de 2020, siendo una de las cuarentenas más largas del mundo. Adicionalmente, con la llegada del segundo y tercer pico de fallecimientos y contagios, se tomaron decisiones gubernamentales nacionales y locales para mitigar su impacto.

Sobre pronóstico del comportamiento futuro del coronavirus se han aplicado diferentes modelos predictivos basados en el Composite Monte Carlo (3), detallados para estimar el curso de la pandemia (4), utilizando el pronóstico de datos oficiales (5), matemáticos, series de tiempo (6), funciones polinomiales (7) y ARIMA (8), entre otros.

En Colombia se han utilizado modelos de predicción como el SIR -S: población susceptible, I: población infectada, R: población recuperada- $(9,10)$, funciones polinomiales $(11)$, análogos de ARIMA como el modelo de Brown (12) y el SEIR -S: población susceptible, E: expuesto, I: población infectada, R: población recuperada- (13), entre otros.

La estimación de modelos brinda la posibilidad de predecir el comportamiento del virus y anticipar algunas implicaciones de la pandemia bajo ciertos parámetros, pudiendo usarse para hacer planes de respuestas futuras. El abordaje de la pandemia desde la epidemiología, implica el uso de modelos matemáticos y econométricos los cuales sirven para la toma de decisiones (14) . Con estos se pueden construir funciones lineales con tasa de variación aritmética o exponenciales con tasas no constantes de variación (15). La regresión polinomial se considera un caso de la regresión lineal múltiple, donde se busca determinar el mejor polinomio que represente los datos de un conjunto de puntos $(16,17)$.

Las curvas de tendencia basadas en regresión polinómica siguen de manera más fiel los datos que otros tipos de modelos matemáticos. Esto se debe a que se recoje la información diariamente, lo que permite predecir más adecuadamente la tendencia. A pesar de ello las ecuaciones polinómicas presentan una gran variación. La mayor utilidad de la regresión polinomial puede encontrarse en la evaluación de las medidas tomadas durante el periodo de cuarentena (11).

El análisis de regresión polinomial busca probar la existencia de una relación entre la variable independiente $\mathrm{x}$ y la variable dependiente $\mathrm{y}$, modelado como un polinomio de enésimo grado en $\mathrm{x}$. La regresión polinomial se ajusta a una relación no lineal entre el valor de x y la media 
condicional correspondiente de $\mathrm{y}$, denotada $\mathrm{E}(\mathrm{y} \mid \mathrm{x})$. Aunque la regresión polinomial ajusta un modelo no lineal a los datos, como problema de estimación estadística, es lineal en los parámetros desconocidos que se calculan a partir de los datos (18).

Este estudio aporta el desarrollo de modelos polinomiales para determinar el comportamiento del crecimiento de la curva de contagios y muertes por COVID-19 en Colombia, a través de una aplicación sencilla de Excel, de acceso desde cualquier computador en sitios remotos y sin modelos predictivos complejos.

\section{Métodos}

Diseño del estudio. Este estudio es de enfoque cuantitativo, exploratorio, no experimental y de corte transversal. Para su desarrollo se recopiló la información de contagios y fallecidos del Instituto Nacional de Salud de Colombia, de carácter público, disponible en la página web https://www.ins.gov.co/noticias/paginas/coronavirus.aspx. Se tomó dato por dato informado diariamente para contagios y fallecimientos por espacio de un año y un mes; todos los casos informados incluidos los importados. La información se registró en hojas de cálculo de Excel donde se modelaron diariamente los polinomios. Posteriormente, se construyó un modelo de regresión polinomial sobre los datos obtenidos usando polinomios de orden 1 hasta 6.

Análisis estadístico. Las variables fueron analizadas en el programa Microsoft Excel 2019®, en el que también se calcularon las correspondientes regresiones polinomiales de tendencia futura con su respectivo $\mathrm{R}^{2}$. Se calcularon los $\mathrm{R}^{2}$ ajustados de manera indirecta ya que Excel no los calcula; de igual manera se elaboraron las gráficas de tendencia de fallecimientos y contagios diarios y acumulados. Se modeló la tendencia proyectando los datos para los siguientes días a través de la opción de línea de tendencia del programa Excel tomado en cuenta las gráficas acumuladas. La regresión polinomial está dada por la ecuación (1):

$$
Y=B o+B 1 X+B 2 X 2+B 3 X 3+B 4 X 4+\cdots+B n X n+E
$$

Espacio temporal. Los datos considerados en el análisis de regresión fueron tomados desde el 06 de marzo de 2020, fecha en la cual se reportó el primer contagio en el país, hasta el 10 de abril de 2021.

\section{Resultados}

De la información diaria recolectada se evaluaron seis funciones polinomiales. El polinomio con mayor nivel de determinación es el de grado 6 según el $\mathrm{R}^{2}$ y $\mathrm{R}^{2}$ ajustado para los casos confirmados de contagiados y fallecidos, de acuerdo con los resultados presentados en las tablas 1 y 2. 
Tabla 1 Polinomios para fallecidos y confirmados contagiados (desde 06 de marzo de 2020 a 10 de abril de 2021)

\begin{tabular}{|c|c|c|c|c|c|c|}
\hline \multirow[b]{2}{*}{ Orden } & \multicolumn{3}{|c|}{ Fallecidos } & \multicolumn{3}{|c|}{ Confirmados contagiados } \\
\hline & Polinomio & $\mathbf{R}^{2}$ & $\begin{array}{c}\mathbf{R}^{2} \\
\text { ajustado }\end{array}$ & Polinomio & $\mathbf{R}^{2}$ & $\underset{\text { ajustado }}{\mathbf{R}^{2}}$ \\
\hline 1 & $y=0,5107 x+68,315$ & 0,2763 & 0,2744 & $y=24,464 x+1716,2$ & 0,3640 & 0,3640 \\
\hline 2 & $\begin{array}{r}y=-0,0049 x 2+2,4756 x \\
-65,249\end{array}$ & 0,5341 & 0,5317 & $\begin{array}{l}y=-0,1807 x^{2}+94,951 x- \\
2877,2\end{array}$ & 0,5647 & 0,5658 \\
\hline 3 & $\begin{array}{l}y=3 E-06 x^{3}-0,0066 x^{2}+ \\
2,7482 x-74,874\end{array}$ & 0,535 & 0,5313 & $\begin{array}{l}y=-0,0004 x^{3}+0,0694 x^{2} \\
+55,875 x-1599,1\end{array}$ & 0,5746 & 0,5768 \\
\hline 4 & $\begin{array}{l}\mathrm{y} \\
=5 \mathrm{E}-09 \times 4-1 \mathrm{E}-06 \times 3 \\
-0,0056 \times 2+2,6545 \mathrm{x} \\
-72,793\end{array}$ & 0,535 & 0,5301 & $\begin{array}{l}y=4 E-06 x^{4}-0,0033 x^{3}+ \\
0,7853 x^{2}-6,3529 x- \\
371,62\end{array}$ & 0,5813 & 0,5845 \\
\hline 5 & $\begin{array}{l}\mathrm{y} \\
=-3 \mathrm{E}-09 \mathrm{x} 5+3 \mathrm{E}-06 \mathrm{x} 4 \\
-0,0009 \times 3+0,1372 \mathrm{x} 2 \\
-5,8807 \mathrm{x}+60,676\end{array}$ & 0,5987 & 0,5934 & $\begin{array}{l}y=1 E-09 x^{5}+3 E-06 x^{4}- \\
0,0029 x^{3}+0,729 x^{2}- \\
3,1985 x-413,37\end{array}$ & 0,5802 & 0,5845 \\
\hline 6 & $\begin{array}{l}y=3 E-11 x^{6}-4 E-08 x^{5}+ \\
2 E-05 x^{4}-0,0043 x^{3}+0,4822 x^{2} \\
-20,666 x+235,84\end{array}$ & 0,6693 & 0,6641 & $\begin{array}{l}y=1 E-09 x^{6}-2 E-06 x^{5}+ \\
0,0007 x^{4}-0,1509 x^{3}+ \\
15,207 x^{2}-572,22 x+ \\
5006,2\end{array}$ & 0,6788 & 0,6830 \\
\hline
\end{tabular}

Fuente: elaboración propia. 
Tabla 2 Polinomios para fallecidos y confirmados contagiados acumulados (desde 06 de marzo de 2020 a 10 de abril de 2021)

\begin{tabular}{|c|c|c|c|c|c|c|}
\hline \multirow[b]{2}{*}{ Orden } & \multicolumn{3}{|c|}{ Fallecidos } & \multicolumn{3}{|c|}{ Confirmados contagiados } \\
\hline & Polinomio & $\mathbf{R}^{2}$ & $\begin{array}{l}\mathbf{R}^{2} \\
\text { ajustado }\end{array}$ & Polinomio & $\mathbf{R}^{2}$ & $\begin{array}{l}\mathbf{R}^{2} \\
\text { ajustado }\end{array}$ \\
\hline 1 & $y=149,36 x$ & 0,96050 & 0,96050 & $y=5528,7 x$ & 0,9444 & 0,94426 \\
\hline 2 & $y=0,3611 x^{2}+43,858 x$ & 0,98191 & 0,98191 & $\begin{array}{l}y=13,528 x^{2}+2134,5 x- \\
144822\end{array}$ & 0,9884 & 0,98834 \\
\hline 3 & $\begin{array}{l}y=-0,0017 x^{3}+1,2242 x^{2} \\
-56,835 x-20,501\end{array}$ & 0,98466 & 0,99466 & $\begin{array}{l}y=-0,0692 x^{3}+54,036 x^{2} \\
-4192,7 x+62133\end{array}$ & 0,9966 & 0,99657 \\
\hline 4 & $\begin{array}{l}y=5 E-06 x^{4}-0,0059 x^{3}+ \\
2,2755 x^{2}-148,22 x+ \\
1782,1\end{array}$ & 0,99466 & 0,99535 & $\begin{array}{l}y \\
=-0,0001 x 4 \\
+0,0144 x 3+33,044 x 2 \\
-2368 x+26139\end{array}$ & 0,9967 & 0,99667 \\
\hline 5 & $\begin{array}{l}y=-4 E-08 x^{5}+4 E-05 x^{4} \\
-0,0187 x^{3}+4,1576 x^{2}- \\
253,62 x+3176,9\end{array}$ & 0,99535 & 0,99564 & $\begin{array}{l}y=-1 E-06 x^{5}+0,0013 x^{4} \\
-0,4731 x^{3}+104,48 x^{2}- \\
6368,8 x+79085\end{array}$ & 0,9971 & 0,997096 \\
\hline 6 & $\begin{array}{l}y=-1 E-09 x^{6}+1 E-06 x^{5} \\
-0,0005 x^{4}+0,0991 x^{3}- \\
7,3682 x^{2}+199,37 x- \\
1137,5\end{array}$ & 0,9984 & 0,99837 & $\begin{array}{l}y=-2 E-08 x^{6}+2 E-05 x^{5} \\
-0,01 x^{4}+1,8867 x^{3}- \\
126,35 x^{2}+2703,4 x- \\
7322,8\end{array}$ & 0,9978 & 0,99777 \\
\hline
\end{tabular}

Fuente: elaboración propia.

La regresión que arroja un $\mathrm{R}^{2}$ más bajo es la del polinomio con grado 1 (regresión lineal simple) para casos confirmados de contagiados y fallecidos. En la figura 1 se presenta la curva generada para fallecidos y en la figura 2 para casos de contagiados confirmados. 


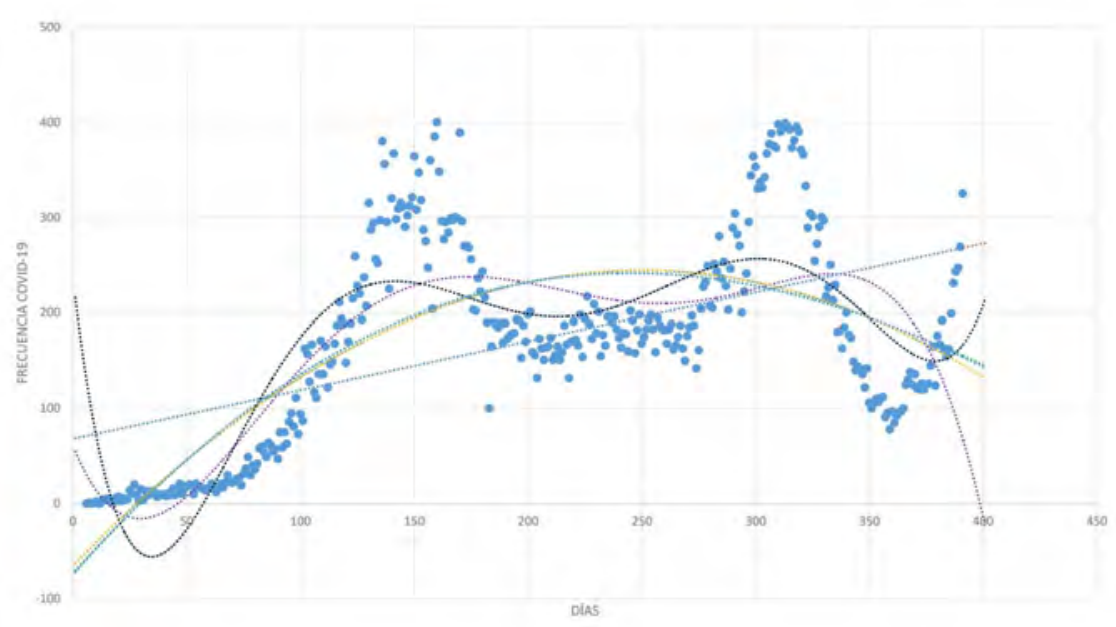

Figura 1 Tendencia de los fallecidos por COVID-19 (desde 06 de marzo de 2020 a 10 de abril de 2021)

Fuente: elaboración propia.

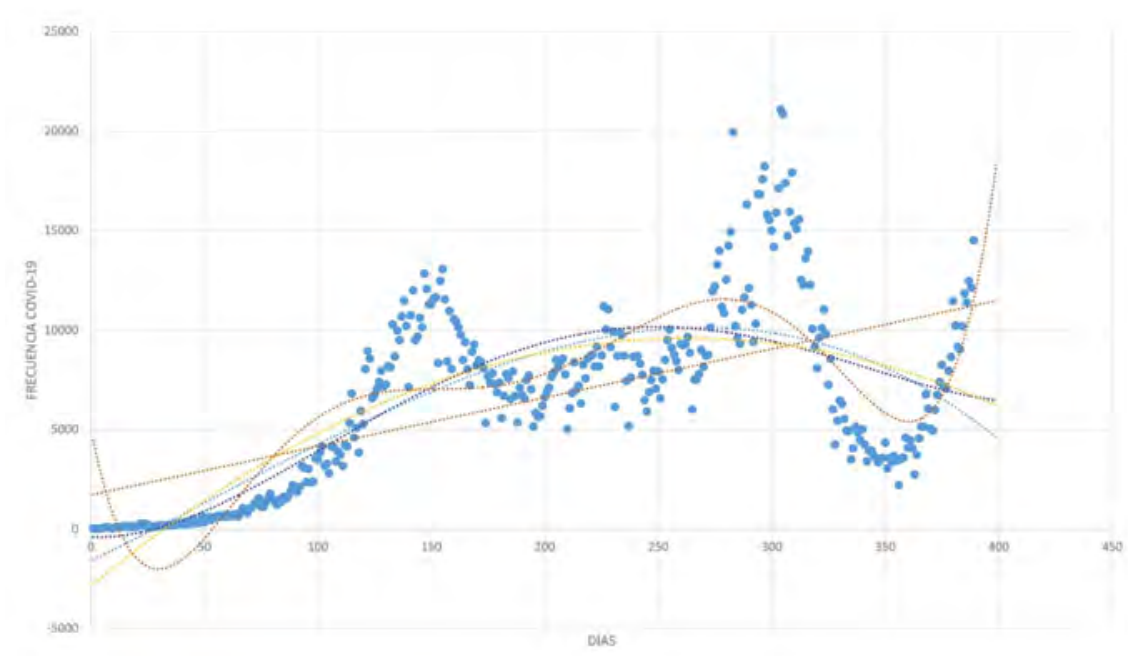

Figura 2 Tendencia de los confirmados contagiados por COVID-19 (desde 06 de marzo de 2020 a 10 de abril de 2021) Fuente: elaboración propia.

De igual manera se realizaron los cálculos para los datos acumulados de contagiados confirmados y fallecidos. Con estos se realizaron las predicciones teniendo en cuenta que la serie presenta una tendencia lineal como se puede apreciar en las figuras 3 y 4 . 
Ariel Emilio Cortés Martínez / Carmen Elisa Becerra Huertas

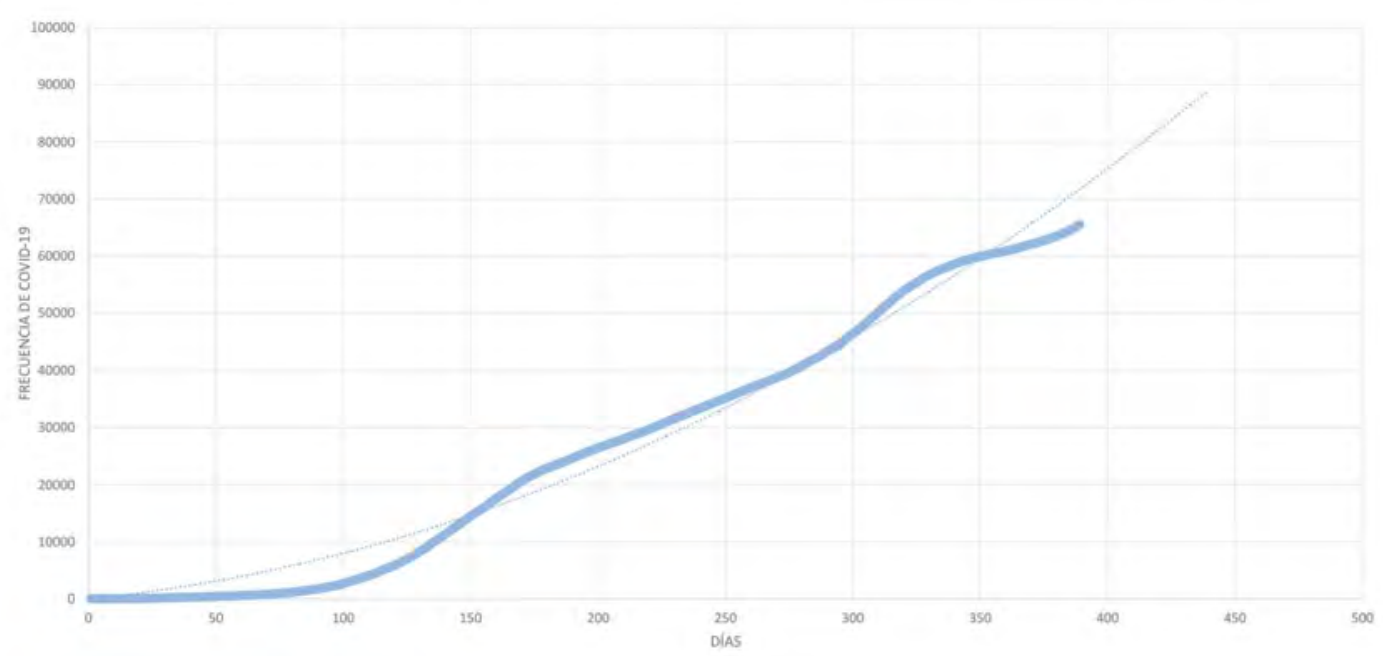

Figura 3 Tendencia de los fallecidos acumulados por

COVID-19 (desde 06 de marzo de 2020 a 10 de abril de 2021)

Fuente: elaboración propia.

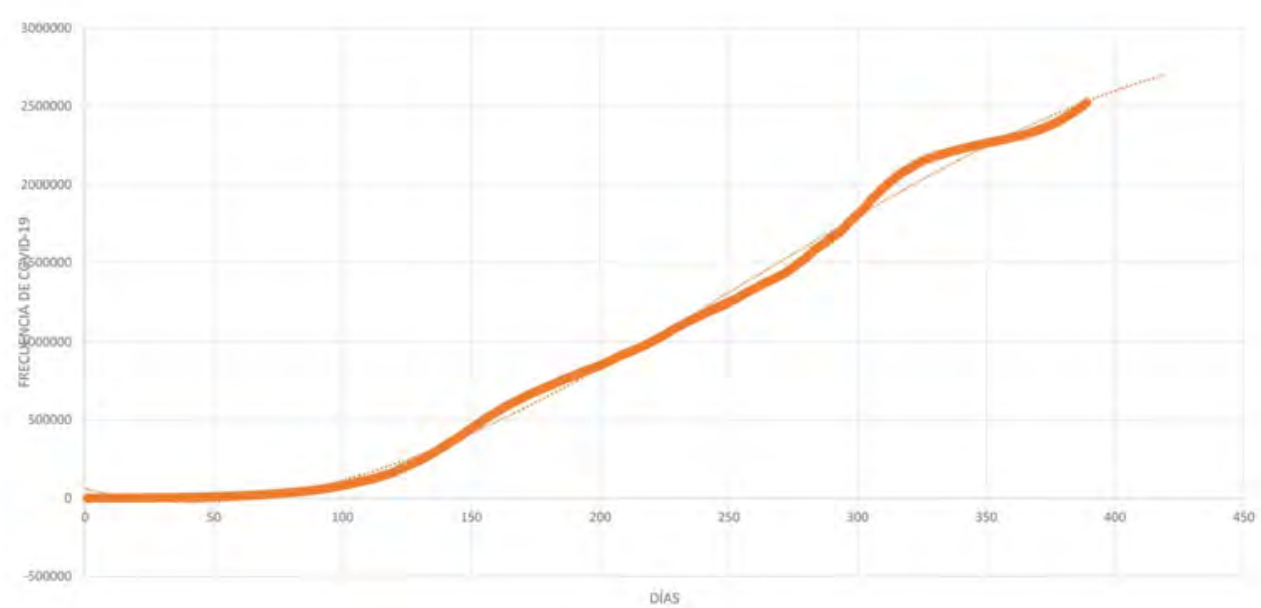

Figura 4 Tendencia de los confimados contagiados acumulados por COVID-19 (desde 06 de marzo de 2020 a 10 de abril de 2021) Fuente: elaboración propia.

La función para confimados contagiados acumulados tiene un $R^{2}$ de 0,9966 expresada mediante la ecuación (2): 


$$
y=-0,0692 x 3+54,036 x 2-4192,7 x+62133
$$

La función para fallecidos acumulados tiene un $\mathrm{R}^{2}$ de 0,982 expresada mediante la ecuación (3):

$$
y=0,3611 x 2+43,858 x
$$

En la tabla 3 se muestan las predicciones de contagiados y de fallecidos a las fechas 20 de abril y 1 de junio de 2021.

Tabla 3 Estimaciones para acumulados fallecidos y contagiados confirmados

\begin{tabular}{|c|c|c|c|}
\hline Estudio & Día & Fecha & Casos \\
\hline \multirow{2}{*}{$\begin{array}{l}\text { Contagiados } \\
\text { confirmados }\end{array}$} & 400 & Abril 20 de 2021 & 2.602 .013 \\
\cline { 2 - 4 } & 442 & Junio 01 de 2021 & 2.790 .167 \\
\hline \multirow{2}{*}{ Fallecidos } & 400 & Abril 20 de 2021 & 76.120 \\
\cline { 2 - 4 } & 442 & Junio 01 de 2021 & 114.690 \\
\hline
\end{tabular}

Fuente: elaboración propia.

\section{Discusión}

Dado que cuando se trata de una pandemia las tendencias pueden dar mejor explicación que las frecuencias absolutas, este estudio reconoce la importancia de la transversalidad. Compartimos que las técnicas de regresión lineal y polinomial son herramientas matemáticas que pueden ser utilizadas para predecir y modelar el comportamiento de un conjunto de datos (11).

En Colombia ya se han realizado estudios que utilizan funciones polinomiales, los cuales plantean la volatilidad de los polinomios con valores futuros. Las ecuaciones polinómicas son más útiles para modelar el comportamiento de la curva del COVID-19 hasta el día de captura de los datos y pueden ser usadas para determinar el impacto de las medidas gubernamentales en un periodo determinado de tiempo, lo cual compartimos.

Cuando se realizan modelos logísticos, es necesario ajustar diariamente la curva desde el comienzo de la infección, porque cada día se obtienen diferentes valores para los parámetros. Aunque el modelo logístico parece ser el más razonable, es probable que la forma de la 
curva cambie debido a los efectos exógenos (19). Dichos efectos exógenos como los puntos de acceso para nuevos contagiados, las acciones del gobierno nacional y los gobiernos territoriales, la estocasticidad en las primeras dinámicas, las heterogeneidades en los patrones de contacto, la variación espacial, la incertidumbre en los parámetros epidemiológicos claves, el distanciamiento social que aplana la curva y el riesgo de resurgimiento al levantar las restricciones, pueden hacer que el tiempo y el ancho del pico sean inciertos (20). A lo anterior se puede sumar el efecto de la vacunación que inició en Colombia el 17 de febrero de 2021.

Una estimación y una predicción basadas en un modelo de pronóstico inconveniente pueden afectar la resiliencia de los sistemas de salud teniendo consecuencias indeseables. En este sentido, los modelos de regresión polinomiales parecen ser los mejores (21). Otro estudio (22) plantea que el modelo exponencial-polinomial, muestra que la aproximación y la predicción realizadas estiman el valor del número acumulado de casos reportados por el Estado colombiano, con un error pequeño.

Como en todo estudio basado en datos tan volátiles e inciertos como el número de positivos reportados, los resultados deben tomarse con cautela (23).

En cuanto a las limitaciones, se encuentra que el programa Microsoft Excel 2019 permite obtener tendencias asociadas a la regresión polinomial. Sin embargo, tiene como limitantes que la estimación de la tendencia polinomial automática solo aborda polinomios hasta de orden 6 $\mathrm{y}$ no es posible obtener de manera directa el valor del error cuadrático medio ni el $\mathrm{R}^{2}$ ajustado. De igual manera, el Gobierno Nacional informó a través del Ministerio de Salud de un ajuste del 17,2\% para los datos informados en el año 2020, de manera que la cifra de la mortalidad aumentó en 7.402 casos sobrepasando los 50.000. El dato que se tenía de fallecidos a 31 de diciembre de 2020 era de 43.116 , por lo que esto afecta el modelamiento de los datos generando inconvenientes en los cálculos.

\section{Conclusiones}

El programa Excel que se encuentra al alcance de cualquier profesional y que no tiene mayor complejidad, permite construir modelos de fácil acceso como los desarrollados a partir de funciones polinomiales, de manera diaria, las cuales son útiles para la vigilancia de eventos de salud pública. Lo anterior facilicita la toma de decisiones oportunas por parte de las autoridades sanitarias locales, a pesar de sus limitaciones como no calcular de manera directa el $\mathrm{R}^{2}$ ajustado.

\section{Consideraciones éticas}

Para todos los fines, los datos utilizados son anónimos. La investigación es de carácter institucional y está aprobada en el Programa en Administración de Salud de la Facultad de Ciencias Económicas y Administrativas. Las opiniones expresadas en este manuscrito 
son responsabilidad de los autores y no reflejan necesariamente el criterio o política de las universidades.

\section{Contribución de los autores}

A. Cortés realizó los análisis de los datos y redacción del artículo. C. Becerra realizó la revisión de literatura, recolección diaria de información y redacción del artículo.

\section{Financiación}

Los autores manifiestan que el estudio fue financiado por la Pontificia Universidad Javeriana y la Universidad Colegio Mayor de Cundinamarca.

\section{Conflictos de interés}

Los autores manifiestan no tener conflicto de interés.

\section{Agradecimientos}

Los autores agradecen a los estudiantes Sergio Barbón, María Becerra y Katherine Rojas del semillero de investigación de organizaciones en salud por la recolección de la información en la matriz de Excel para el desarrollo del artículo.

\section{Referencias}

1. Long-quan L, Huang T, Wang Y, Wang Z, Liang Y, Huang T, et al. COVID-19 patients' clinical characteristics, discharge rate, and fatality rate of meta-analysis. J Medic Virol. 2020;92(6):577-583. http://dx.doi.org/10.1002/jmv.25757

2. Paredes Y, Chipia J. COVID-19: estadística de morbi-mortalidad en Venezuela. GICOS: Revista del Grupo de Investigaciones en Comunidad y Salud. 2020;5(2):47-66. http://erevistas.saber.ula.ve/ index,php/gicos/article/view/16120

3. Fong S, Li G, Dey N, Crespo R, Herrera-Viedma E. Finding an accurate early forecasting model from small dataset: A case of 2019-nCoV novel coronavirus outbreak. Preprint. 2020;2003.10776. http:/ /dx.doi.org/ijimai.2020.02.002

4. Bertozzi A, Franco E, Mohler G, Short M, Sledge D. The challenges of modeling and forecasting the spread of COVID-19. Preprint. 2020;2004.04741. http://dx.doi.org/10.1073/pnas.2006520117

5. Li L, Yang Z, Dang Z, Meng C, Huang J, Meng H, et al. Propagation analysis and prediction of the COVID-19. Infectious Disease Modelling. 2020;5:282-292. https://doi.org/10.1016/j.idm.2020.03.0 02

6. Mizumoto K, Chowell G. Transmission potential of the novel coronavirus (COVID-19) onboard the Diamond Princess Cruises Ship, 2020. Infectious Disease Modelling. 2020;5:264-270. https://doi.o $\mathrm{rg} / 10.1016 / \mathrm{j} . \mathrm{idm} .2020 .02 .003$ 
7. Hernandez-Matamoros A, Fujita H, Hayashi T, Perez-Meana H. Forecasting of COVID19 per regions using ARIMA models and polynomial functions. Applied Soft Computing. 2020;96:106610. http:// dx.doi.org/10.1016/j.asoc. 2020.106610

8. Benvenuto D, Giovanetti M, Vassallo L, Angeletti S, Ciccozzi M. Application of the ARIMA model on the COVID-2019 epidemic dataset. Data in brief. 2020;(29):105340. http://dx.doi.org/10.1016/j.dib .2020 .105340

9. Manrique F, González-Chordá V, Gutiérrez O, Tellez C, Herrera-Amaya G. Modelo SIR de la pandemia de Covid-19 en Colombia. Revista Salud Pública Universidad Nacional. 2020;22:1-9. http://dx.doi. org/10.15446/rsap.v22n2.85977

10. Bizet N, Pena D. Time-dependent and time-independent SIR models applied to the COVID-19 outbreak in Argentina, Brazil, Colombia, Mexico and South Africa. 2006.12479. arXiv preprint arXiv:2006.12479. https://arxiv.org/pdf/2006.12479.pdf

11. Golondrino G, Muñoz WML. Aplicación de la regresión polinomial para la caracterización de la curva del COVID-19 en Colombia, mediante técnicas de machine learning. Investigación e Innovación en Ingenierías. 2020;8(2);87-105. http://dx.doi.org/10.17081/invinno.8.2.4103

12. Pinzón J. Proyección de la propagación del COVID-19 en Colombia. Revista Med de la Facultad de Medicina. 2020;28(1):11-20. http://dx.doi.org/10.18359/rmed.4702

13. Arango-Londoño D, Ortega-Lenis D, Muñoz E, Cuartas D, Caicedo D, Mena J, et al. Predicciones de un modelo SEIR para casos de COVID-19 en Cali, Colombia. Rev Salud Públ. 2020;22:1-6. https: //doi.org/10.15446/rsap.V22n2.86432

14. Montesinos-López, Hernández-Suárez. Modelos matemáticos para enfermedades infecciosas. Salud Públ Méx. 2007;49(3):218-226. http://dx.doi.org/10.1590/S0036-36342007000300007

15. Heras, Donado, Pachón. Métodos cuantitativos y análisis epidemiológico en vigilancia. Vigilancia Epidemiológica de Navarra. Madrid: McGraw-Hill Interamericana; 2004.

16. Ferretti L, Wymant C, Kendall M, Zhao L, Nurtay A, Abeler-Dörner L, et al. Quantifying SARS-CoV-2 transmission suggests epidemic control with digital contact tracing. Science. 2020;368(6491). http: //dx.doi.org/10.1126/science.abb6936

17. Kucharski A, Russell T, Diamond C, Liu Y, Edmunds J, Funk S, et al. Early dynamics of transmission and control of COVID-19: A mathematical modelling study. The lancet infectious diseases. 2020;20(5):553-558. http://dx.doi.org/10.1016/S1473-3099(20)30144-4

da Rocha A. Regression Polynomial Analysis of the COVID-19 Epidemics: Some Initial Findings; 2020. http://dx.doi.org/10.2139/ssrn.3636820

19. Avendaño V. Infección del Covid-19 en Colombia. Una comparación de modelos logísticos y exponenciales aplicados a la infección por el virus en Colombia; 2020. https://www.researchgate.net/publication/340092755_Infeccion_del_Covid-19_en_Colombia _Una_comparacion_de_modelos_logisticos_y_exponenciales_aplicad os_a_la_infeccion_por_el_virus_en_Colombia 
20. Martínez J, Vargas A. Covid-19: evolución y estimaciones de las curvas epidémicas; 2020. http://bibliodigitalibd.senado.gob.mx/bitstream/handle/123456789/4877/Reporte\%20TE\%20 78\%20Curvas\%20epidemicas\%20F.pdf?sequence $=1$

21. Gerli A, Centanni S, Miozzo M, Sotgiu G. Predictive models of COVID-19 Related deaths and infections. Int J Tuberc Lung Dis. 2020;24:10. http://dx.doi.org/10.5588/ijtld.20.0196

22. Bravo A, Vera M, Huérfano YK. Modelos matemáticos estimadores de la infección por COVID-19: consideraciones esenciales y proyecciones en Colombia. Rev Salud Public. 2020;22(3):1-7. https:// doi.org/10.15446/rsap.V22n3.87813

23. Cagigal M, Becario F. Modelado y análisis de la evolución de una epidemia vírica mediante filtros de Kalman: el caso de la COVID-19 en España. Depósito de investigación de la Universidad de Sevilla. España: Universidad de Sevilla. 2020. https://idus.us.es/bitstream/handle/11441/94508/Analisis\%20de\%20la\%20evolucion\%20del \%20COVID-19\%20con\%20Filtros\%20de\%20Kalman\%20v1\%20\%2801-04-2020\%29.pdf? sequence $=7 \&$ is Allowed $=$ yLo guarde $\mathrm{pdf}$ 